\title{
The allometric model in chronic myocardial infarction
}

\author{
Maria P Bonomini ${ }^{1 *}$, Pedro D Arini ${ }^{1,2}$, Germán E Gonzalez $^{3}$, Bruno Buchholz ${ }^{3}$ and Max E Valentinuzzi ${ }^{1}$
}

\author{
* Correspondence: pbonomini@fi. \\ uba.ar \\ ${ }^{1}$ Instituto de Ingeniería Biomédica \\ (IIBM), Facultad de Ingeniería (FI), \\ Universidad de Buenos Aires (UBA), \\ Buenos Aires, Argentina \\ Full list of author information is \\ available at the end of the article
}

\begin{abstract}
Background: An allometric relationship between different electrocardiogram (ECG) parameters and infarcted ventricular mass was assessed in a myocardial infarction (MI) model in New Zealand rabbits.

Methods: A total of fifteen animals were used, out of which ten underwent left anterior descending coronary artery ligation to induce infarction (7-35\% area). Myocardial infarction (MI) evolved and stabilized during a three month-period, after which, rabbits were sacrificed and the injured area was histologically confirmed. Right before sacrifice, ECGs were obtained to correlate several of its parameters to the infarcted mass. The latter was normalized after combining data from planimetry measurements and heart weight. The following ECG parameters were studied: RR and $P R$ intervals, $P$-wave duration $\left(\mathrm{P}_{\mathrm{D}}\right)$, QRS duration $\left(\mathrm{QRS} \mathrm{S}_{\mathrm{D}}\right)$ and amplitude $\left(\mathrm{QRS} \mathrm{A}_{\mathrm{A}}\right)$, Q-wave $\left(Q_{A}\right)$, R-wave $\left(R_{A}\right)$ and $S$-wave $\left(S_{A}\right)$ amplitudes, T-wave peak amplitude $\left(T_{A}\right)$, the interval from the peak to the end of the T-wave $\left(T_{P E}\right)$, ST-segment deviation $\left(\mathrm{ST}_{\mathrm{A}}\right), \mathrm{QT}$ interval (QT), corrected QT and JT intervals. Corrected QT was analyzed with different correction formulae, i.e., Bazett $\left(\mathrm{Q} T_{B}\right)$, Framingham $\left(\mathrm{Q} T_{F R A}\right)$, Fridericia $\left(\mathrm{QT}_{\mathrm{FR}}\right)$, Hodge $\left(\mathrm{Q} \mathrm{T}_{\mathrm{HO}}\right)$ and Matsunaga $\left(\mathrm{QT} \mathrm{T}_{\mathrm{MA}}\right)$ and compared thereafter. The former variables and infarcted ventricular mass were then fitted to the allometric equation in terms of deviation from normality, in turn derived after ECGs in 5 healthy rabbits.
\end{abstract}

Results: Six variables ( $J T, \mathrm{QT}_{B}, \mathrm{Q}_{A}, \mathrm{~S}_{A}, \mathrm{~T}_{A}$ and $\mathrm{ST}_{A}$ ) presented statistical differences among leads. QT showed the best allometric fit $(r=0.78)$, followed by $\mathrm{T}_{\mathrm{A}}(r=0.77)$, $\mathrm{ST}_{\mathrm{A}}(r=0.75), \mathrm{QT}_{\text {FRA }}(r=0.72), \mathrm{T}_{\mathrm{PE}}(r=0.69), \mathrm{QT}_{\text {FRI }}(r=0.68)$ and $\mathrm{QT}_{\mathrm{MA}}(r=0.68)$.

Corrected QT's (QT FRA, $Q T_{F R I}$ and $Q T_{M A}$ ) performed worse than the uncorrected counterpart (QT), the former scaling allometrically with similar goodness of fits.

Conclusions: $\mathrm{QT}, \mathrm{T}_{\mathrm{A}}, \mathrm{ST}_{\mathrm{A}}$ and $\mathrm{T}_{\mathrm{PE}}$ could possibly be used to assess infarction extent in an old Ml event through the allometric model as a first approach. Moreover, the $T_{P E}$ also produced a good allometric scaling, leading to the potential existence of promising allometric indexes to diagnose malignant arrhythmias.

Keywords: Allometric law, ECG parameters, Chronic infarction extent, Myocardial infarction model

\section{Background}

In a previous paper [1], we briefly gave the basic idea to apply the allometric concept in electrocardiography, that is, and quoting almost verbatim: «Scaling of many biological processes can be described by the allometric equation $Y=a^{*}\left(B_{\text {mass }}\right)^{b}$, where $Y$ is the biological process, $\mathrm{B}_{\text {mass }}$ the body mass and "a" and "b" are scaling constants. In general, the weights of most individual organs scale as a constant fraction of body mass (i.e., the body

(c) 2012 Bonomini et al.; licensee BioMed Central Ltd. This is an Open Access article distributed under the terms of the Creative Commons Attribution License (http://creativecommons.org/licenses/by/2.0), which permits unrestricted use, distribution, and reproduction in any medium, provided the original work is properly cited. 
mass exponent, "b" equals 1.0). Volume rates, instead (the product of volume and rate), such as cardiac output, ventilation and oxygen uptake, vary with "b" around 0.75. Finally, rates (heart and respiratory rate) scale as " $b$ " close to 0.25 . These emergent patterns provide insights into body-size dependent principles that seem to dictate several aspects of design and function across species among all mammals $[2,3] »$.

Noujaim et al. [4] assumed that the heart behaves as a set of "fractal-like" networks tending to minimize propagation time across the conducting system while ensuring a hemodynamically optimal atrioventricular activation sequence. With the mathematical relationship given above and, subsequently, based on previously published values of PR interval, heart rate, and body masses of 541 mammals, they reported as best fit the equation $\mathrm{PR}=53 \times\left(\mathrm{B}_{\text {mass }}\right)^{0.24}$.

Inspired in the latter report, the following question seems pertinent:

Would a relationship similar to the allometric equation be conceivable, say, between the number of cardiac diseased fibers and any of several ECG parameters when a myocardial infarct (MI) affected the heart?

The objective of this paper tries to find an answer to such question. The Q-wave growth appears as a good candidate because well-known is the fact that normal depolarization suffers with compromised myocardial mass, augmenting the Q-wave and shrinking the R-wave within the QRS complex. Also, additional MI changes include alterations in the T-wave morphology and QRS complex voltages and duration, as well as distortion in intervals such as JT and QT. Many reports confirm this concept, such as Klootwijk, in 1998 [5], Kléber, in 2000 [6], or Balian et al., in 2006 [7], among others. All of these variables and their behaviour on a MI model deserve to be looked at under the allometric light, which seems to maintain interest, especially in general mammalian biology $[3,8]$.

\section{Methods}

\section{Experimental protocol and procedure to myocardial infarction}

An overall of fifteen animals were used in this study. Ten New Zealand rabbits (7 male and 3 female, $1.5-2.5 \mathrm{~kg}$ ) underwent left anterior descendent (LAD) coronary artery ligation to provoke infarction on a varying extent of myocardial tissue. MI evolved and stabilized during a three month-period after which, rabbits were sacrificed and MI histologically confirmed.

Ventricular infarcted mass in grams was calculated after combining data from planimetric measurements and heart weights. The infarcted mass was then normalized to heart weight in order to compare among different rabbit sizes. Normalized ventricular infarcted mass $\left(\mathrm{VIM}_{\mathrm{n}}\right)$ and the ECG parameters mentioned above were, thereafter, fitted to the allometric equation.

This study conformed to the Guide for the Care and Use of Laboratory Animals published by the US National Institutes of Health (NIH Publication No. 85-23, revised 1996).

After anaesthesia with ketamine $(75 \mathrm{mg} / \mathrm{kg})$ and Rompun $(0,75 \mathrm{mg} / \mathrm{kg}$ xylazine) was administered subcutaneously, an endotracheal tube ( $3 \mathrm{~mm}$ inner diameter) was placed to mechanically ventilate the rabbits with room air using a Harvard respirator $(25 \mathrm{ml}$, 34-38 cycles/min). Immediately after, $5 \%$ dextrose solution $(3 \mathrm{ml} / \mathrm{min})$ was infused 
through a flexible catheter placed at the marginal vein of one of the ears. Using this venous access throughout the surgical procedure, anaesthesia was maintained by applying additional doses of ketamine and sodium thyopental as needed during the surgical procedure. A left thoracotomy on the fourth intercostal space and a pericardiectomy were performed to expose the heart. Thereafter, a lateral or posterolateral coronary artery branch of the LAD coronary artery was ligated using a curved needle and 6.0 silk thread [9]. The appearance of regional paleness confirmed ventricular wall ischemia. Finally, the thorax was closed with linen thread keeping the order of the different anatomical parts. At the end of the surgical procedure, the animals were placed in a quiet environment for their recovery. Antiobiotic therapy was administered and animals were closely observed during the first 24 hours. Afterwards, animals were placed in individual cages up to the finalization of a three month period.

For the control group, 5 healthy rabbits underwent the above mentioned anaesthetic protocol and ECG recordings were obtained. Analogously to the MI group, the three standard limb leads were recorded.

\section{Histology and planimetry}

To assess infarct size, Masson's Trichrome (MT) staining was carried out, while infarction was corroborated by Hematoxiline-Eosine (HE). Once excised, hearts were perfused with $10 \%$ formaldehyde during 5 min for fixation, remaining in that solution for at least 72 hs. Four millimeter slices were cut out from apex to base and then processed for paraffin embedding and staining. After the latter, fibrotic tissue turned blue, so differentiating itself from healthy tissue, while HE allowed morphological recognition of necrotic tissue.

The stained slices were scanned and planimetric measurements were performed using an image software (Image Pro-Plus 4.5). The area mean percent occupied by the scar tissue at each ventricular level estimated the infarct size [10,11]; these values were expressed as percentage of the total ventricular area while normalized ventricular infarcted mass $\left(\mathrm{VIM}_{\mathrm{n}}\right)$ was calculated as follows:

$$
\mathrm{VIM}_{\mathrm{n}}=\operatorname{PIM}(\%) * \mathrm{VM}(\mathrm{gr}) / \mathrm{HW}(\mathrm{gr})
$$

where PIM: planimetric infarcted area (\% of the total ventricular area); VM: ventricular mass and HW: heart weight.

\section{ECG acquisition and preprocessing}

Rabbits were heparinized (500 U/Kg IV) 10 minutes before anesthesia by intramuscular injection of ketamine $(35 \mathrm{mg} / \mathrm{Kg})$ and lidocaine $(5 \mathrm{mg} / \mathrm{Kg})$. All animals were in sinus rhythm at the time of ECG recordings (frontal leads I, II, and III).

ECG data were recorded using instrumentation amplifiers with a gain factor of 1000 and a bandwidth of $0.05-150 \mathrm{~Hz}$. The signals were digitalized at a sampling rate $\mathrm{F}_{\mathrm{s}}=500 \mathrm{~Hz}$ and 12-bit resolution using a digital acquisition board (Lab PC+, National Instruments, Austin, TX, USA). When necessary, a band-stop filter was used to remove $50-\mathrm{Hz}$. All of the data were acquired and monitored using customized software made in $\mathrm{C}++$. 
QRS detection was based on methods described by Hamilton and Tompkins in 1986 [12]. All the ectopic or aberrant beats were automatically rejected by the computer. If needed, baseline corrections were performed by cubic spline interpolation [13].

We computed the running signal average of 30 beats. The template for signal averaging in each ECG channel were created, thereafter, by aligning 30 beats with $98 \%$ cross-correlated QRS waveforms using the predefined window and the R-wave peak as the trigger point while, at the same time, correcting for fiducial point jitter. Two rejected beats were the accepted limit on the computation of each final template beat.

\section{ECG delineation}

ECG delineation was accomplished on the template obtained as explained in section "ECG acquisition and preprocessing". The start and end of the QRS complex were determined by searches on each side of the R-wave for regions where the slope (dV/dt) fell to sufficiently low values. Isoelectric level was taken as the median of all data values preceding the start of the QRS. For the P-wave, we used a modified version of the algorithm described by [14], which is based on finding the peaks and valleys of a 9-point derivative signal. In case of the T-wave delineation, a search was made for the first significant peak of either sign, starting from a point after the end of the QRS. If a suitable peak was found, a straight line was adjusted by least squares from the peak to the tail of the T-wave. The intersection of this line with the isoelectric line was accepted as the T-wave end. ST-segment deviation was defined as the ECG amplitude $50 \mathrm{~ms}$ after QRS onset [15].

For all ECG templates, the PR interval, $\mathrm{P}$-wave $\left(\mathrm{P}_{\mathrm{D}}\right)$ and $\mathrm{QRS}$ duration $\left(\mathrm{QRS} \mathrm{D}_{\mathrm{D}}\right), \mathrm{QRS}$ amplitude $\left(\mathrm{QRS}_{\mathrm{A}}\right)$, $\mathrm{T}$-wave peak amplitude $\left(\mathrm{T}_{\mathrm{A}}\right)$, $\mathrm{ST}$-segment deviation $\left(\mathrm{ST}_{\mathrm{A}}\right)$, the time from the peak to the end of the T-wave $\left(\mathrm{T}_{\mathrm{PE}}\right), \mathrm{QT}$ interval, corrected $\mathrm{QT}$ interval $\left(\mathrm{QT}_{\mathrm{C}}\right)$ and $\mathrm{JT}$ intervals (JT) were calculated. Below the details of the QT corrections are given according to different formulae. All parameters are graphically represented in Figure 1.

\section{QT $\mathrm{C}$ Formulae}

The following QT correction formulae were tested:

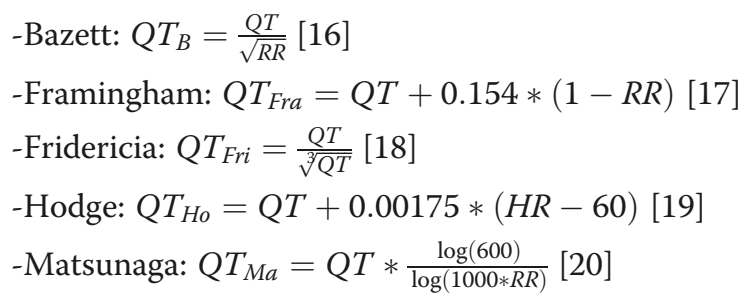

where $\mathrm{HR}=$ heart rate in $\mathrm{bpm}, \mathrm{RR}=\mathrm{RR}$ interval in $\mathrm{s}, \mathrm{QT}=\mathrm{QT}$ duration in $\mathrm{s}, \mathrm{QT}_{\mathrm{C}}=$ corrected QT interval in s.

\section{Allometric model - Mathematical setting}

Allometry, in general biology, measures the relative growth of a part in relation to the whole living organism. The term was first used by Snell, in 1891 [9], to express the mass of a mammal's brain as a function of the body mass. The growth velocity of a component $y$ is related to the growth velocity of another component (or the whole organism) $x$ in a constant way. This was clearly described by von Bertalanffy in 1957 [10]. 


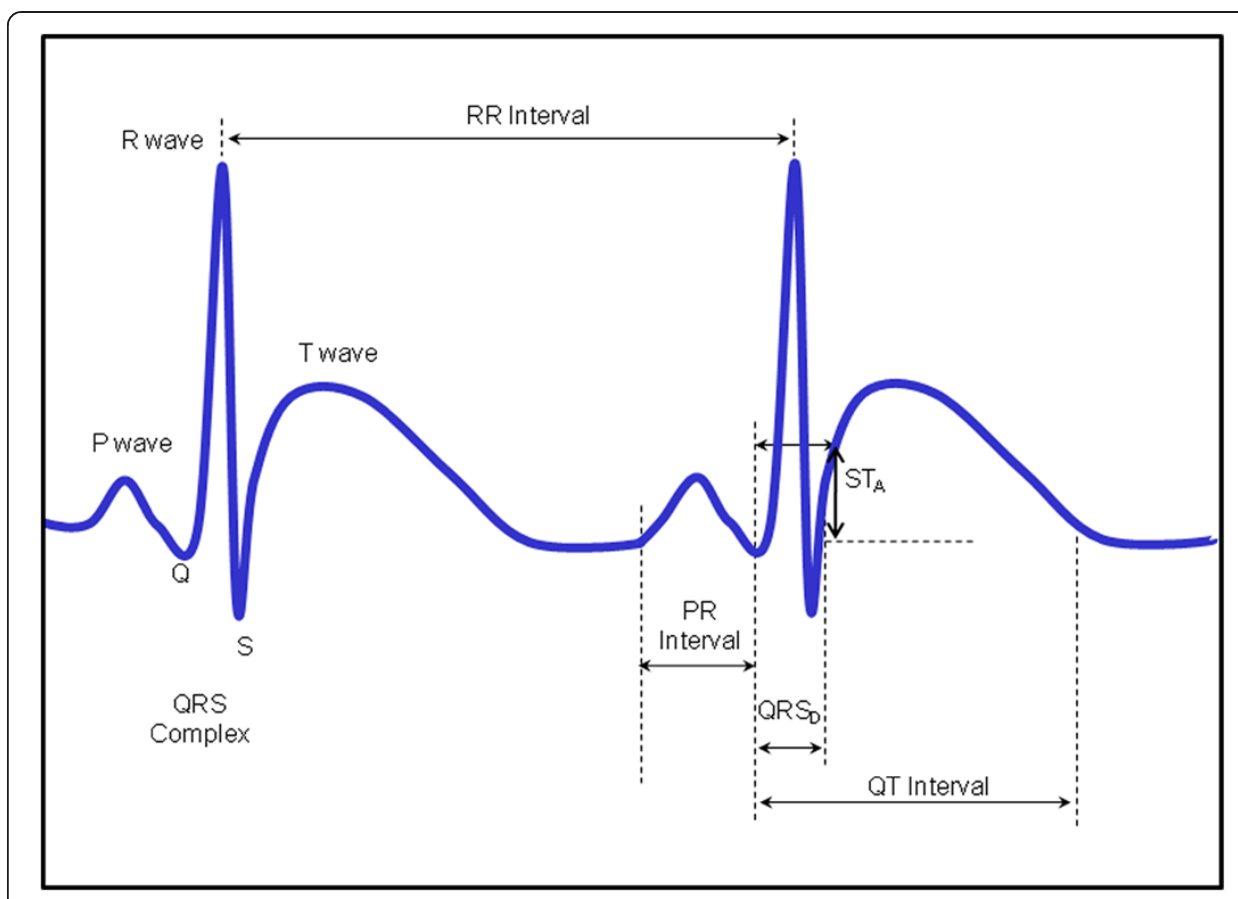

Figure 1 ECG measurements. Schematic representation of ECG waves, intervals and segments analyzed in this work.

Thus, the relative rate of change of a given event $y$ is proportional to the relative rate of change of body mass or body weight $x$, i.e.,

$$
\frac{d y / d t}{y}=B^{*} \frac{d x / d t}{x}
$$

After integration and some easy algebraic manipulation, equation (1) becomes

$$
\ln y=A+B^{*} \ln x,
$$

or

$$
y=A^{*} x^{B}
$$

Originally, $y$ was the weight of an organ (heart, stomach, other) and $x$ was body weight or mass. The parameters $A$ and $B$ require numerical estimation by an appropriate procedure usually using empirical information. By the same token, let us hypothesize that the electrocardiographic parameters follow a relationship with the number of ventricular fibers $\left(N_{F}\right)$, formally equal to (2), i.e.,

$$
E C G_{\text {param }}=a^{*}\left(N_{F}\right)^{B}
$$

Moreover, in a pathology context, let us relate the "deviation from normality" in terms of ECG with the "deviation from normality" in terms of diseased fibers, modifying the model as in (4),

$$
E C G_{D}-E C G_{N}=a^{*}\left(N_{D F}\right)^{\beta}
$$

Hence, $y$ in equation (2) is replaced by $\left(\mathrm{ECG}_{D}-\mathrm{ECG}_{N}\right)$ in (3), and $N_{D F}$ in the latter takes the place of $x$ in the former. After all, the number of diseased cardiac fibers 
(ischemic or infarcted or both) are part of the cardiac mass. Besides, since the relationship between diseased fibers and infarcted mass is straightforward, it sounds sensible to state that,

$$
N_{D F}=\gamma^{*} V I M_{n}
$$

Hence,

$$
E C G_{D}-E C G_{N}\left(\gamma^{*} V I M_{n}\right)^{\beta}
$$

After taking logarithms of both sides, the latter equation becomes $\log \left(E C G_{D}-E C G_{N}\right)=\left(\log a+\beta^{*} \log \gamma\right)+\beta^{*}\left(\log V I M_{n}\right)$, which can be reduced to

$$
\log \left(E C G_{D}-E C G_{N}\right)=\delta+\beta^{*}\left(\log V I M_{n}\right)
$$

where $\delta=\log \alpha+\beta^{*} \log \gamma, \mathrm{VIM}_{\mathrm{n}}$ : $\log$ (normalized ventricular infarcted mass) and $\mathrm{ECG}_{D}$ and $\mathrm{ECG}_{N}$ are the diseased and healthy counterparts of the following ECG parameters; $\mathrm{ST}_{\mathrm{A}}: \log (\mid \mathrm{ST}$ deviation $\mid)$ in $\mathrm{mV} ; \mathrm{Q}_{\mathrm{A}}: \log (\mid \mathrm{Q}$-wave amplitude $\mid)$ in $\mathrm{mV} ; \mathrm{S}_{\mathrm{A}}: \log (\mid \mathrm{S}$-wave amplitude $\mid)$ in $\mathrm{mV} ; \mathrm{R}_{\mathrm{A}}: \log (\mid \mathrm{R}$-wave amplitude $\mid)$ in $\mathrm{mV} ; \mathrm{QRS}_{\mathrm{A}}: \log (|\mathrm{Q}|+|\mathrm{R}|+|\mathrm{S}|)$ in $\mathrm{mV} ; \mathrm{T}_{\mathrm{A}}: \log \left(\mathrm{T}\right.$-wave peak amplitude) in $\mathrm{mV}, \mathrm{T}_{\mathrm{PE}}: \log (\mathrm{T}$-wave time from peak to end); $\mathrm{QRS}_{\mathrm{D}}: \log (\mathrm{QRS}$ width);.QT: $\log (\mathrm{QT}$ interval); QTc: $\log (\mathrm{QT}$ interval corrected by different formulae); RR: $\log \left(\mathrm{RR}\right.$ interval); $\mathrm{PR}: \log \left(\mathrm{PR}\right.$ interval); $\mathrm{P}_{\mathrm{D}}$ : $\log (\mathrm{P}$ duration). Linear regressions were carried out to obtain numerical values for the intercept $\delta$ and the slope $\beta$ in the log-log plot.

\section{Statistical analysis}

Data are expressed as mean \pm standard deviation (Mean \pm SD). To assess statistical significance, leads I, II and III were compared among them. When data did not pass the D’Agostino \& Pearson normality test, a non-parametric Kruskall-Wallis test was applied and the Dunn's post test carried out. Otherwise, one-way ANOVA and Bonferroni post-test were calculated.

\section{Results}

\section{ECG parameters}

ECG parameters were consistent with MI, showing wider and morphologically diverse QRS complexes. QRS voltages suffered in MI, presenting a significant decrease in leads II and III. Due to flattening of the T-wave, $\mathrm{ST}_{\mathrm{A}}$ resulted very small in Leads II and III, but presented a rounded pattern in Lead I. QRS $\mathrm{D}_{\mathrm{D}}$ was similar in every lead. Neither significant RR nor PR interval changes were seen. $P_{D}$ was longer in lead II, even though no statistical significance was found. Similarly, $\mathrm{T}_{\mathrm{PE}}$ increased in Lead II but failed to be statistically significant. Lengthening of the JT interval was originated by a flattening of the T-wave, which is much more rounded in the physiological state and offered statistical significance in lead II with respect to the other two leads. The T-wave peak voltage significantly decreased to even become negative in leads II and III, representing the typical T-wave inversion described in chronic infarction [21,22]. Table 1 displays the values for the ECG timerelated parameters described above along with the statistical significance values $(p<0.05)$; so does Table 2 for the ECG amplitude-related parameters.

QT interval did not show significant differences among leads. On the other hand, among all the correction formulae tested (Bazett, Framingham, Friderica, Hodges and 
Table 1 Electrocardiographic time-related parameters (Mean \pm SD) from a 3 month-MI rabbit evolution

\begin{tabular}{lllllll}
\hline & RR $(\mathbf{m s})$ & PR $(\mathbf{m s})$ & $\mathbf{P}_{\mathbf{D}}$ (ms) & QRS $_{\mathbf{D}}$ (ms) & JT (ms) & T $_{\mathbf{P E}}$ (ms) \\
\hline L1 & $365 \pm 92$ & $71 \pm 8$ & $29 \pm 7$ & $45 \pm 6$ & $120 \pm 21$ & $59 \pm 23$ \\
L2 & $397 \pm 99$ & $76 \pm 8$ & $32 \pm 6$ & $45 \pm 4$ & $161 \pm 35^{*}$ & $74 \pm 25$ \\
L3 & $394 \pm 108$ & $72 \pm 9$ & $24 \pm 6$ & $41 \pm 4$ & $127 \pm 26$ & $59 \pm 24$ \\
\hline
\end{tabular}

L1: lead I, L2: lead II, L3: lead III. Statistical significance: ${ }^{*} p<0.05$, Lead II against Leads I and III.

Matsunaga), the only one that led to statistical significance was Bazett's formula, with a clear differentiation from Leads II and III. Table 3 summarizes the QT and QT corrected values for every lead.

\section{Allometric fits}

In general terms, 7 out of 18 measures presented a clear allometric scaling with VIMn Lead I contained 4 out of 7 such parameters $\left(\mathrm{ST}_{\mathrm{A}}, \mathrm{QT}_{\mathrm{FRA}}, \mathrm{QT}_{\mathrm{FRI}}\right.$ and $\left.\mathrm{QT}_{\mathrm{MA}}\right)$, followed by Lead III with 2 (QT and $\mathrm{T}_{\mathrm{PE}}$ ) and Lead II $\left(\mathrm{T}_{\mathrm{A}}\right)$ with only one. This lead preference for each parameter explains the way data are presented, on a lead separated-basis. The variable that best fitted the allometric equation was QT $(r=0.78)$ followed by $\mathrm{T}_{\mathrm{A}}$ $(r=0.77), \mathrm{ST}_{\mathrm{A}}(r=0.75), \mathrm{QT}_{\mathrm{FRA}}(\mathrm{r}=0.72), \mathrm{T}_{\mathrm{PE}}(r=0.69), \mathrm{QT}_{\mathrm{FRI}}(r=0.68)$ and $\mathrm{QT}_{\mathrm{MA}}$ $(r=0.68)$, all of these with statistical significance $(\mathrm{p}<0.05)$. It is worth noting that $\mathrm{T}_{\mathrm{A}}$ presented a negative allometric fit with $\operatorname{VIM}_{n}(\beta=-2.474 \pm 0.7736)$, meaning that the value of T-voltage decreased as VIM $_{n}$ increased. A similar fit was found for the Q-wave amplitude $(\beta=-0.6352 \pm 0.2849)$, even though it failed to account for statistical significance $(\mathrm{p}=0.0610)$. On the contrary, $\mathrm{S}_{\mathrm{A}}$ increased along with $\mathrm{VIM}_{\mathrm{n}}$ producing a positive slope $(\beta=1.202 \pm 0.6668)$ in the allometric equation. This opposite trend would explain the change in morphology of QRS complexes, shifting from a QR to an RS pattern. $\mathrm{ST}_{\mathrm{A}}$ deviation in Lead I displayed a good linear regression with $\mathrm{VIM}_{\mathrm{n}}$ in a $\log$-log representation. The slope for this regression turned up to be positive, leading to an $\mathrm{ST}_{\mathrm{A}}$ increase with infarct size. On the other hand, $\mathrm{T}_{\mathrm{PE}}$ in Lead III also increased with $\mathrm{VIM}_{\mathrm{n}}$ reflecting important changes in T-wave morphology, mostly due to the flattening of the $\mathrm{T}$-wave, which is normally rounded in rabbits. Figure 2 displays the allometric scaling of the mentioned variables $\mathrm{QT}, \mathrm{T}_{\mathrm{A}}, \mathrm{T}_{\mathrm{PE}}$ and $\mathrm{ST}_{\mathrm{A}}$. Adjustments are shown as well as 95\% confidence limits (broken lines).

QT corrections presented allometric fits with $\mathrm{VIM}_{\mathrm{n}}$ as well, and were analyzed in a different graph (Figure 3) for clarity sake. In lead I, the Framingham, Matsunaga and Fridericia formulae corrections for heart rate allometrically scaled to $\mathrm{VIM}_{\mathrm{n}}$. Accordingly, all the corrected QT intervals displayed a slope smaller than one, near 0.25 , as

Table 2 Electrocardiographic amplitude-related parameters (Mean \pm SD) from a 3 monthMI rabbit evolution

\begin{tabular}{lllllll}
\hline & $\mathbf{Q}_{\mathbf{A}}(\mathbf{m V})$ & $\mathbf{R}_{\mathbf{A}}(\mathbf{m V})$ & $\mathbf{S}_{\mathbf{A}}(\mathbf{m V})$ & $\mathbf{Q R S}_{\mathbf{A}}(\mathbf{m V})$ & $\mathbf{S T}_{\mathbf{A}}(\mathbf{m V})$ & $\mathbf{T}_{\mathbf{A}}(\mathbf{m V})$ \\
\hline L1 & $-0.50 \pm 0.33^{*}$ & $0.21 \pm 0.23$ & $-0.12 \pm 0.36^{*}$ & $0.83 \pm 0.92$ & $0.08 \pm 0.04^{*}$ & $0.10 \pm 0.12^{*}$ \\
L2 & $-0.29 \pm 0.23$ & $0.22 \pm 0.16$ & $0.01 \pm 0.05$ & $0.52 \pm 0.44$ & $0.01 \pm 0.05$ & $-0.03 \pm 0.09$ \\
L3 & $-0.12 \pm 0.15$ & $0.37 \pm 0.24$ & $-0.02 \pm 0.03$ & $0.51 \pm 0.42$ & $-0.02 \pm 0.03$ & $-0.05 \pm 0.07$ \\
\hline
\end{tabular}

L1: lead I, L2: lead II, L3: lead III. Statistical significance: ${ }^{*} p<0.05$, Lead I against Lead III. 
Table 3 Corrected and uncorrected QT intervals (Mean \pm SD) from a 3 month-MI rabbit evolution

\begin{tabular}{lllllll}
\hline & QT (ms) & QT $_{\mathbf{B}}(\mathbf{m s})$ & $\mathbf{Q T}_{\mathrm{FRA}}(\mathbf{m s})$ & $\mathbf{Q T}_{\mathrm{FRI}}(\mathbf{m s})$ & $\mathbf{Q T}_{\mathrm{HO}}(\mathbf{m s})$ & QT $_{\mathrm{MA}}$ (ms) \\
\hline L1 & $182 \pm 24$ & $296 \pm 25$ & $272 \pm 16$ & $242 \pm 23$ & $359 \pm 50$ & $189 \pm 20$ \\
L2 & $187 \pm 35$ & $293 \pm 42$ & $265 \pm 19$ & $235 \pm 28$ & $350 \pm 54$ & $184 \pm 25$ \\
L3 & $169 \pm 29$ & $272 \pm 28^{* 2}$ & $262 \pm 20$ & $231 \pm 25$ & $347 \pm 57$ & $182 \pm 25$ \\
\hline
\end{tabular}

L1: lead I, L2: lead II, L3: lead III. QT $T_{F R A}$ : Framingham correction, $\mathrm{QT}_{\mathrm{FR}}$ : Fridericia correction, $\mathrm{QT}_{\mathrm{MA}}$ : Matsunaga correction, $\mathrm{QT}_{\text {Hо: }}$ Hodge correction. Statistical significance: ${ }^{*} p<0.05$, Lead III versus Lead II.

cited in the bibliography [2-4], being the uncorrected QT the only one that deviated from this pattern $(\beta=3.464 \pm 1.072)$.

The remaining variables, $\mathrm{RR}, \mathrm{PR}, \mathrm{P}_{\mathrm{D}}, \mathrm{QRS}_{\mathrm{D}}, \mathrm{JT}, \mathrm{QT}_{\mathrm{HO}}, \mathrm{QT}_{\mathrm{B}}, \mathrm{QRS}_{\mathrm{A}}$ and $\mathrm{Q}_{\mathrm{A}}, \mathrm{R}_{\mathrm{A}}$ and $\mathrm{S}_{\mathrm{A}}$ did not present a fit with a good statistical significance $(\mathrm{p}>0.05)$. Moreover, they kept almost constant for all MI extents (data not shown).

A complete description of the allometric fits can be found in Table 4, where all parameters together with the slope $\beta$, the origin intercept $\delta$, the goodness of fit $r$ and the statistical significance $p$-value are shown. Each parameter was chosen from the lead at which the best allometric fit was produced.

\section{Discussion}

Findings of the present study

An allometric study was presented here in order to analyze the allometric scaling of different ECG parameters with respect to the infarction extent in a chronic experimental

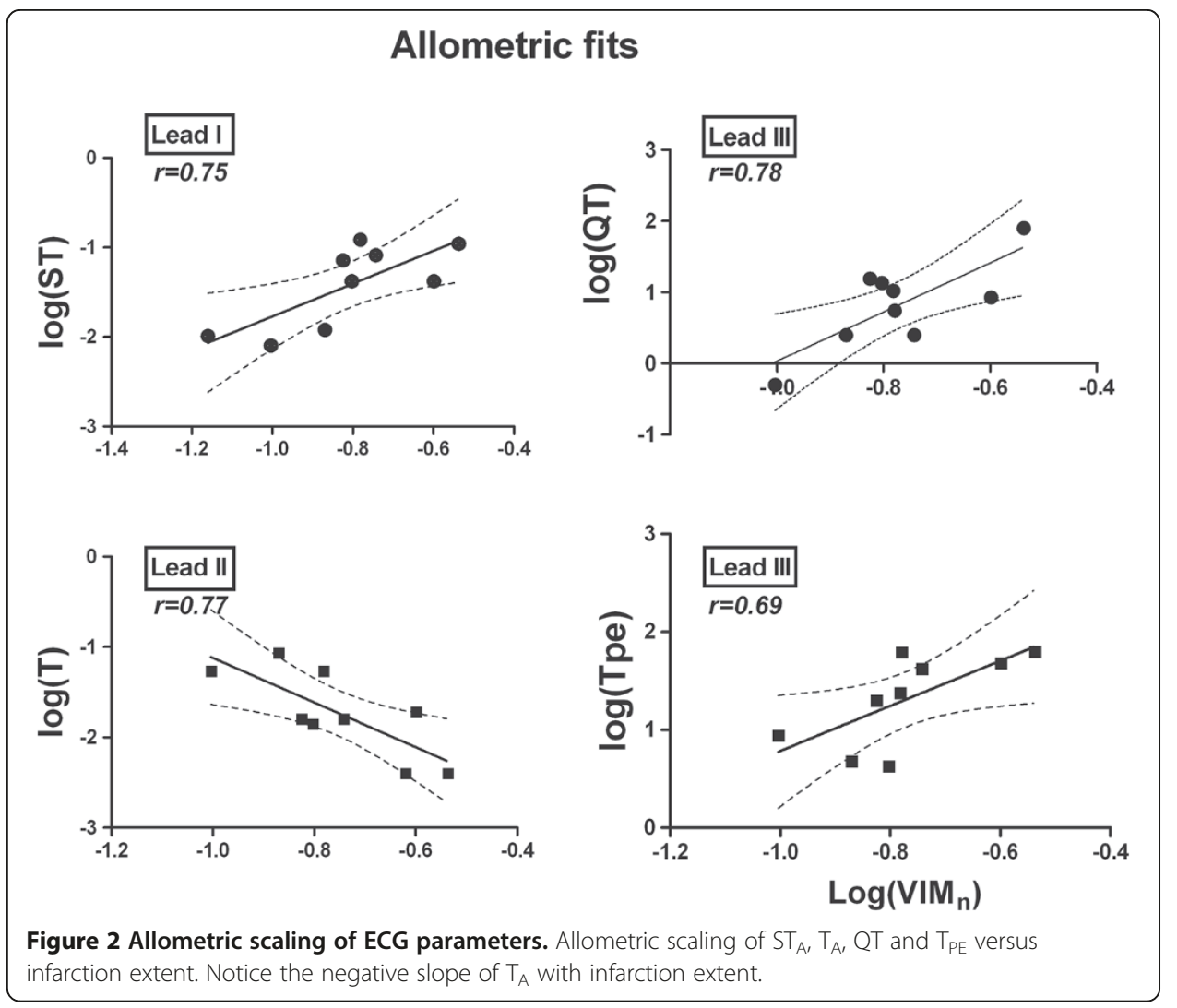




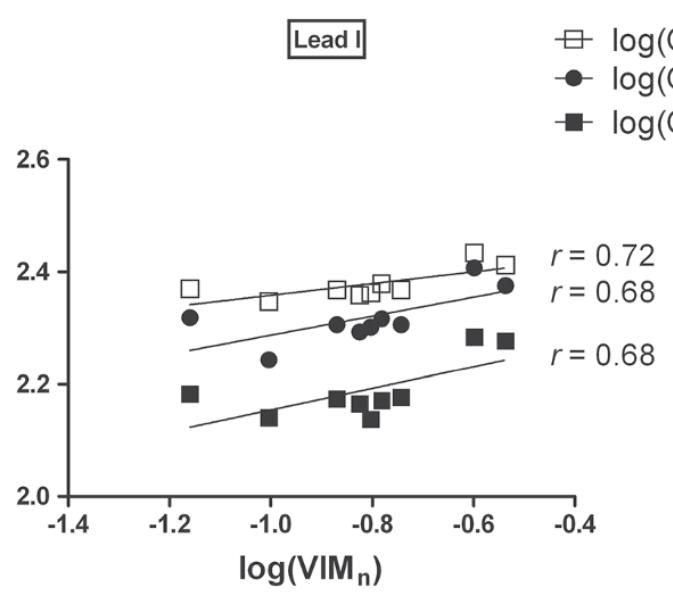

Figure 3 Allometric scaling of QT and QTc parameters. Corrected QT allometric fits. QTfra: Framingham correction formula, QTfri: Fridericia correction formula, QTma: Matsunaga correction formula.

MI rabbit model. The idea behind this work was to find ECG data to estimate myocardial injured area as a first, simple and inexpensive step before resorting to more complex or invasive technologies, such as magnetic resonance imaging or radioscopic procedures. The chief findings of this study were as follows:

Table 4 Linear regressions of all ECG parameters for a 3 month-MI rabbit evolution grouped by leads

\begin{tabular}{|c|c|c|c|c|c|}
\hline Lead & Parameter & $\beta($ Mean $\pm S D)$ & $\delta($ Mean $\pm S D)$ & $r$ & $p$-value \\
\hline L3 & QT & $3.464 \pm 1.072$ & $3.494 \pm 0.8387$ & 0.78 & 0.0145 \\
\hline L2 & $\mathrm{T}_{\mathrm{A}}$ & $-2.474 \pm 0.7736$ & $-3.593 \pm 0.5926$ & 0.77 & 0.0151 \\
\hline L1 & $\mathrm{ST}_{\mathrm{A}}$ & $1.832 \pm 0.5997$ & $0.06115 \pm 0.4994$ & 0.75 & 0.0185 \\
\hline L1 & $\mathrm{QT}_{\text {FRA }}$ & $0.1042 \pm 0.03840$ & $2.462 \pm 0.03198$ & 0.72 & 0.0300 \\
\hline L3 & $\mathrm{T}_{\mathrm{PE}}$ & $2.303 \pm 0.9132$ & $3.087 \pm 0.7142$ & 0.69 & 0.0397 \\
\hline L1 & $\mathrm{QT}_{\mathrm{FRI}}$ & $0.1691 \pm 0.06926$ & $2.456 \pm 0.05769$ & 0.68 & 0.0447 \\
\hline L1 & $\mathrm{QT}_{\mathrm{MA}}$ & $0.1918 \pm 0.07858$ & $2.346 \pm 0.06545$ & 0.68 & 0.0447 \\
\hline L1 & $\mathrm{QT}_{\mathrm{B}}$ & $0.1384 \pm 0.0617$ & $2.531 \pm 0.05142$ & 0.64 & 0.0600 \\
\hline L3 & $\mathrm{Q}_{\mathrm{A}}$ & $-0.6352 \pm 0.2849$ & $-0.8878 \pm 0.2228$ & 0.64 & 0.0610 \\
\hline L3 & $\mathrm{S}_{\mathrm{A}}$ & $1.202 \pm 0.6668$ & $0.03635 \pm 0.5215$ & 0.55 & 0.1145 \\
\hline L2 & $P_{D}$ & $1.011 \pm 0.5893$ & $1.510 \pm 0.4514$ & 0.54 & 0.1299 \\
\hline L1 & $\mathrm{QRS}_{\mathrm{A}}$ & $1.210 \pm 0.7190$ & $0.2376 \pm 0.5988$ & 0.53 & 0.1362 \\
\hline L3 & $\mathrm{QRS}_{\mathrm{D}}$ & $0.5699 \pm 0.3995$ & $1.566 \pm 0.3124$ & 0.47 & 0.1968 \\
\hline L1 & $\mathrm{RR}$ & $0.5025 \pm 0.3669$ & $2.675 \pm 0.3056$ & 0.45 & 0.2131 \\
\hline L2 & $P R$ & $-0.7553 \pm 0.7338$ & $0.3425 \pm 0.5621$ & 0.36 & 0.3376 \\
\hline L1 & $\mathrm{R}_{\mathrm{A}}$ & $0.7621 \pm 0.7972$ & $0.09869 \pm 0.6639$ & 0.34 & 0.3709 \\
\hline L3 & JT & $0.5296 \pm 0.6854$ & $1.821 \pm 0.5360$ & 0.26 & 0.4650 \\
\hline L3 & $\mathrm{QT}_{\mathrm{HO}}$ & $0.1448 \pm 0.3730$ & $2.350 \pm 0.2917$ & 0.14 & 0.7095 \\
\hline
\end{tabular}

$\beta$ : slope of linear regression, $\delta$ : $Y$-intercept when $\mathrm{X}=0$ of linear regression, $r$ : correlation coefficient, $p$-value: statistical significance of linear fit. L1: Lead I, L2: Lead II, L3: Lead III, QT FRA: Framingham correction formula, QT MA: Matsunaga correction formula, $\mathrm{QT}_{\mathrm{B}}$ : Bazett correction formula, $\mathrm{QT}_{\mathrm{FRl}}$ : Fridericia correction formula, $\mathrm{QT}_{\mathrm{HO}}$ : Hodges correction formula $\mathrm{QT}$ : QT interval, $\mathrm{T}_{\mathrm{A}}$ : amplitude of T-wave, $\mathrm{ST}_{\mathrm{A}}$ : amplitude of $\mathrm{ST}$ segment deviation, $\mathrm{T}_{\mathrm{PE}}$ : time from peak to end of the Twave, $\mathrm{Q}_{\mathrm{A}}$ : amplitude of Q-wave, $\mathrm{S}_{\mathrm{A}}$ : amplitude of $\mathrm{S}$-wave, $\mathrm{R}_{\mathrm{A}}$ : amplitude of $\mathrm{R}$-wave, $\mathrm{QRS}_{\mathrm{A}}$ : amplitude of $\mathrm{QRS}$ complex, $\mathrm{QRS}_{\mathrm{D}}$ : duration of QRS complex, RR: RR interval, PR: PR interval, JT: JT interval. 
1- Six variables (JT, $\mathrm{QT}_{\mathrm{B}}, \mathrm{Q}_{\mathrm{A}}, \mathrm{S}_{\mathrm{A}}, \mathrm{T}_{\mathrm{A}}$ and $\mathrm{ST}_{\mathrm{A}}$ ) showed statistical differences among leads. Lead I versus Lead III contained 5 out of 6 of these statistical differences $\left(\mathrm{QT}_{\mathrm{B}}, \mathrm{Q}_{\mathrm{A}}, \mathrm{S}_{\mathrm{A}}, \mathrm{T}_{\mathrm{A}}\right.$ and $\left.\mathrm{ST}_{\mathrm{A}}\right)$, while JT presented differences in Lead II with respect to Lead I. Most of these variables turned out to be amplitude-related $\left(Q_{A}, S_{A}\right.$, $\mathrm{T}_{\mathrm{A}}$ and $\mathrm{ST}_{\mathrm{A}}$ ) and, probably, the lead with the most evident changes was related to infarct localization.

2- Seven out of eighteen parameters presented a significant allometric fit $(\mathrm{p}<0.05)$. Lead I accounted for the majority of them $\left(\mathrm{ST}_{\mathrm{A}}, \mathrm{QT}_{\mathrm{FRA}}, \mathrm{QT}_{\mathrm{FRI}}, \mathrm{QT}_{\mathrm{MA}}\right)$, followed by Lead III ( $\left.\mathrm{QT}, \mathrm{T}_{\mathrm{PE}}\right)$ and Lead II $\left(\mathrm{T}_{\mathrm{A}}\right)$. See Table 4 , where it can also be seen that $\mathrm{QT}_{\mathrm{B}}$ and $\mathrm{Q}_{\mathrm{A}}$ showed borderline significances and still with a good fit.

3- The variables that best adjusted to the allometric equation were $\mathrm{QT}, \mathrm{T}_{\mathrm{A}}$ and $\mathrm{S}_{\mathrm{TA}}$ with a goodness of fit enough to assess infarction size within a sensitive range $(r=0.78, r=0.77, r=0.75$, respectively.

\section{Significance and interpretation of the data}

Ventricular changes found during the chronic MI state can be split into ventricular depolarization and repolarization changes.

\section{Ventricular Depolarization Changes}

In chronic MI hearts, QRS morphologies changed [21,22]. Even though not statistical significant, a negative Q-voltage slope and a positive S-voltage slope in the allometric equation suggest a shift from the QR to the RS morphology.

Surprisingly, $\mathrm{QRS}_{\mathrm{D}}$ did not fit well the allometric equation, as it would have been expected. We cannot find a good explanation for such behaviour. Evidence refers to the prognostic importance of QRS duration in acute and chronic myocardial infarction $[23,24]$; hence, further investigation would be needed.

\section{Ventricular Repolarization Changes}

The T-wave showed a change in morphology, too, becoming flat instead of rounded. Moreover, negative T-wave morphologies appeared, mostly in lead II and III. These changes forced the JT interval to be longer and $\mathrm{T}_{\mathrm{PE}}$ to be longer as well as a smaller $\mathrm{T}_{\mathrm{A}}$. On the other hand, $\mathrm{QT}$ interval and $\mathrm{T}_{\mathrm{PE}}$ presented a good allometric scaling. It is tempting to link this observation with the fact that increased arrhythmogenesis is related to MI hearts, as reported elsewhere [25-32].

The JT-interval could be considered as a total ventricular repolarization process. In our study, such interval was increased in MI, which might indicate a differential lengthening or shortening of the action potential duration (APD) in some myocardial areas, so reflecting the range of times at which action potentials end and, therefore, it would be an expression of APD heterogeneity [33]. However, JT showed a bad allometric scaling and, at least based on this study, we could not establish the allometric behaviour of this parameter.

Uncorrected QT offered the best fit, in agreement with the literature on QT and ischaemia. With transmural ischaemia, the T-wave axis shifts in the opposite direction towards the region of epicardial involvement, being often a sign of infarction. Such Twaves are tall and peaked, frequently with long QT intervals [34]. These changes may assist in the localization of ischaemia and also in the quantification of the injury size. It 
is worth noting that the slope of all corrected QT where about 0.25 , similar to those found in the literature related to allometry; the uncorrected QT was the only one out of this pattern $(\beta=3.464 \pm 1.072)[2,4]$.

Surprisingly, $\mathrm{ST}_{\mathrm{A}}$ deviation in Lead I displayed a good linear regression with $\mathrm{VIM}_{\mathrm{n}}$ in a $\log$-log representation. This fact was opposite to our expectations since in humans, ST deviation tends to normalize in chronic MI. Nevertheless, ST-segment in rodents (especially in rats, and less markedly in rabbits) shows a different behaviour in the physiological case [35]. Thus it is plausible to think that these differences will still hold for the pathological cases.

In addition, someone may think that a multilead approach would be a good methodology to measure the parameters. However, in our case, the number of leads was only three and we think that is not enough number to guarantee that kind of analysis. On top of that, we did not have a criterion to select one or the other lead.

Finally, it should be underlined that the reference set of normal data, even though they appear at first sight as a good idea (see equation \#4), did not significantly changed the results when using a direct relationship such as

$$
E C G_{\text {param }}=a *\left(N_{D F}\right)^{\beta}
$$

from which numerical results are not shown in this report (see also above, Findings of the present study, item 3 ).

\section{Study implications and future work}

The first half of the T-wave is mainly related to epicardial APD, whereas the second half is to the endocardial and mid-myocardial APD [36,37]. As a result, at the peak of the $\mathrm{T}$-wave the transmural voltage gradient reaches the maximum and, therefore, the descending limb represents the extent of transmural dispersion of ventricular repolarization. Therefore, great is the importance of $\mathrm{T}_{\mathrm{PE}}$ producing a good allometric scaling, leading to a new and attractive future work, which is the search of allometric indexes for diagnosing malignant arrhythmias.

$\mathrm{QT}, \mathrm{T}_{\mathrm{A}}$ and $\mathrm{ST}_{\mathrm{A}}$ showed the strongest allometric behavior. This implies that chronic MI extents could be roughly assessed by feeding the allometric equation with any of these parameters. Whether one outstands from the others or not and hence, whether the allometric equation should be fed with any of these parameters individually or collectively with any combination of them, remains open.

Another consideration is pertinent regarding MI localization. Data presented in this work is valid for anteroseptal injury only. Thus, the search of electrocardiographic allometric scaling on different MI localizations would complete this study.

\section{Study limitations}

In the analysis of several electrocardiographic parameters, most of the ECG time-related and amplitude-related measurements have been taken into account. Nevertheless, other approaches for QT correction should be explored, like the individual correction suggested by Malik [38] or the correction for animals carried out by Valentinuzzi [39]. On the other hand, the relations found here are strictly related to infarcts originated from LAD coronary artery ligation, not covering other MI localizations. Besides, a wider 
range of infarction extent would be desirable, allometrically speaking, but not possible due to the extremely low survival rates at MI extents greater than $40 \%$.

\section{Conclusions}

The objectives of this paper were met, since ECG parameters related to depolarization and repolarization phases showed a certain degree of adjustment to the allometric equation, some of them rather good. The parameters that best allometrically scaled were $\mathrm{QT}, \mathrm{T}_{\mathrm{A}}$ and $\mathrm{ST}_{\mathrm{A}}$, with a goodness of fit enough to assess infarction size within an acceptable range. Besides, the $\mathrm{T}_{\mathrm{PE}}$ produced a good allometric scaling, leading to the potential existence of promising indexes to diagnose malignant arrhythmias.

Competing interests

The authors declare that they have no competing interests.

\section{Acknowledgements}

Partially supported by PIP \#538, from CONICET (Consejo Nacional de Investigaciones Científicas y Técnicas). All three authors are CONICET Career Investigators.

\section{Author details}

${ }^{1}$ Instituto de Ingeniería Biomédica (IIBM), Facultad de Ingeniería (FI), Universidad de Buenos Aires (UBA), Buenos Aires, Argentina. ${ }^{2}$ Instituto Argentino de Matemática, "Alberto P. Calderón", CONICET, Buenos Aires, Argentina. ${ }^{3}$ Instituto de Fisiopatología Cardiovascular, Facultad de Medicina, Universidad de Buenos Aires (UBA), Buenos Aires, Argentina.

\section{Authors' contributions}

MPB carried out the animal experiments and drafted the manuscript. MPB, PDA and MEV made key contributions to the conception and design, analysis and interpretation of data, and drafting of the manuscript. GEG and BB run the reference series. All authors read and approved the final manuscript.

Received: 15 February 2012 Accepted: 11 May 2012

Published: 11 May 2012

\section{References}

1. Bonomini MP, Arini PD, Valentinuzzi ME: Probability of ventricular fibrillation: Allometric model based on the ST deviation. Biomed Eng Online 2011, 10:8.

2. Lindstedt SL, Schaeffer PJ: Use of allometry in predicting anatomical and physiological parameters of mammals. Lab Anim 2002, 36:1-19.

3. Martin RD, Genoud M, Hemelrijk CK: Problems of allometric scaling analysis: examples from mammalian reproductive biology. J Experiment Biol 2005, 208:1731-1747.

4. Noujaim SF, Lucca E, Muñoz V, Persaud D, Berenfeld O, Meijler FL, Jalife J: From mouse to whale: A universal scaling relation for the PR interval of the electrocardiogram of mammals. Circulation 2004, 110:2801-2808.

5. Klootwijk APJ: Dynamic computer-assisted ST segment monitoring in patients with acute coronary syndromes: Thesis Dissertation. Rottërdam: Erasmus Universiteit; 1998.

6. Kléber AG: ST-segment elevation in the electrocardiogram: a sign of myocardial ischemia. Cardiovasc Res 2000, 45:111-118.

7. Balian V, Galli M, Marcassa C, Cecchin G, Child M, Barlocco F, Petrucci E, Filippini G, Michi R, Onofri M: Intracoronary ST-segment shift soon after elective percutaneous coronary intervention accurately predicts periprocedural myocardial injury. Circulation 2006, 114:1948-1954.

8. Adler CP, Costabel U: Cell number in human heart in atrophy, hypertrophy, and under the influence of cytostatics. Recent Adv Studies Cardiac Struc Metab 1975, 6:12

9. Podesser B, Wollenek G, Seitelberger R, Siegel H, Wolner E, Firbas W, Tschabitscher M: Epicardial branches of the coronary arteries and their distribution in the rabbit heart: the rabbit heart as a model of regional ischemia. Anat Rec 1997, 247:521-527.

10. Fletcher PJ, Pfeffer JM, Pfeffer MA, Braunwald E: Left ventricular diastolic pressure-volume relations in rats with healed myocardial infarction. Effects on systolic function. Circ Res 1981, 49:618-626.

11. Pfeffer MA, Pfeffer JM, Fishbein MC, Fletcher PJ, Spadaro J, Kloner RA, Braunwald E: Myocardial infarct size and ventricular function in rats. Circ Res 1979, 44:503-512.

12. Hamilton PS, Tompkins WJ: Quantitative investigation of QRS detection rules using the MIT/BIH arrhythmia database. IEEE Trans Biomed Eng BME 1986, 33:1157-1165.

13. Meyer $\mathrm{CR}$, Keiser HN: Electrocardiogram baseline noise estimation and removal using cubic splines and statespace computation techniques. Comput Biomed Res 1977, 10:459-470.

14. Hee-Kyo J, Kwang-Keun K, Sun-Chul H, Lee M-H: A New Algorithm for P-Wave Detection in the ECG Signal. In Book A New Algorithm for P-Wave Detection in the ECG Signal. 1989. (Editor ed.^eds.), vol. 1. pp. 42-43. City; 1989:42-43.

15. Xue Q, Reddy S: Algorithms for computerized QT analysis. J Electrocardiol 1997, 30:181-186.

16. Bazett $\mathrm{H}$ : An analysis of time-relations of electrocardiograms. Heart 1920, vii:17. 
17. Sagie A, et al: An improved method for adjusting the QT interval for heart rate (the Framingham study). Am J Cardiol 1992, 70:4

18. Fridericia L: Die Systolendauer im Elektrokardiogramm bei normalen Menschen und bei Herzkranken. Acta Medica Scandinavica 1920, 53:17.

19. Hodge M: Rate correction of the QT interval. Card Electrophysiol Rev 1997, 3:3.

20. Matsunaga T, Mitsui T, Harada T, Inokuma M, Murano H, Shibutani Y: QT corrected for heart rate and relation between QT and RR intervals in beagle dogs. J Pharmacol Toxicol Methods 1997, 38:201-209.

21. Hu N, Straub CM, Garzarelli AA, Sabey KH, Yockman JW, Bull DA: Ligation of the left circumflex coronary artery with subsequent MRI and histopathology in rabbits. J Am Assoc Lab Animal Sci: JAALAS 2010, 49:838-844.

22. Yu M, Bozek J, Guaraldi M, Kagan M, Azure M, Robinson SP: Cardiac imaging and safety evaluation of BMS747158, a novel PET myocardial perfusion imaging agent, in chronic myocardial compromised rabbits. J Nucl Cardiol: Official Publication of the American Society of Nuclear Cardiology 2010, 17:631-636.

23. Fosbøl EL, Seibaek M, Brendorp B, Møller DV, Ersbøøll M, Torp-Pedersen C, Køber L: Differential prognostic importance of QRS duration in heart failure and acute myocardial infarction associated with left ventricular dysfunction. Eur J Heart Fail 2007, 9:814-823.

24. Brenyo A, Zaręba W: Prognostic significance of QRS duration and morphology. Cardiol J 2011, 18:8-17.

25. Briasoulis A, Agarwal V, Pierce WJ: QT prolongation and torsade de pointes induced by fluoroquinolones: infrequent side effects from commonly used medications. Cardiology 2011, 120:103-110.

26. Han J, Moe GK: Non uniform recovery of excitability in ventricular muscle. Circ Res 1964, 14:44-60.

27. Lubinski A, Kornacewicz-Jach Z, Wnuk-Wojnar AM, Adamus J, Kempa M, Krolak T, Lewicka-Nowak E, Radomski M, Swiatecka G: The terminal portion of the T wave: a new electrocardiographic marker of risk of ventricular arrhythmias. Pacing Clin Electrophysiol 2000, 23:1957-1959.

28. Taggart P, Sutton PMI, Opthof T, Coronel R, Trimlett R, Pugsley W, Kallis P: Transmural repolarization in the left ventricle in humans during normoxia and ischemia. Cardiovasc Res 2001, 50:454-462.

29. Vos MA, Jungschleger GM: Transmural repolarization gradients in vivo. The flukes and falls of the endocardium. Cardiovasc Res 2001, 50:423-425.

30. Nakagawa M, Takahashi N, Watanabe M, Ichinose M, Nobe S, Yonemochi H, Ito M, Saikawa T: Gender differences in ventricular repolarization: Terminal T wave interval was shorter in women than in men. Pacing Clin Electrophysiol 2003, 26:59-64.

31. Kuo CS, Munakata K, Reddy PS, Surawicz B: Characteristics and possible mechanisms of ventricular arrhythmias dependent on dispersion of action potentials. Circulation 1983, 67:1356-1367.

32. Deshmukh A, Ulveling K, Alla V, Abuissa H, Airey K: Prolonged QTc Interval and Torsades de Pointes Induced by Citalopram. Tex Heart Inst J 2012, 39:68-70.

33. Arini PD, Bertrán GC, Valverde ER, Laguna P: T-wave width as an index for quantification of ventricular repolarization dispersion: Evaluation in an isolated rabbit heart model. Biomed Signal Process Contr 2008, 3:67-77.

34. Vandyck-Acquah MPS: Electrocardiographic Background. In Dynamic Electrocardiography. Edited by Malik M, Camm AJ. New York: Blackwell, Futura Publishing: 2004:217-232.

35. Nervonne J, Kass R: Molecular Physiology of Cardiac Repolarization. Physiol Rev 2005, 85:1205-1253.

36. Sicouri S, Antzelevitch C: A subpopulation of cells with unique electrophysiological properties in the deep subepicardium of the canine ventricle. The M Cell Circ Res 1991, 68:1729-1741.

37. Antzelevitch C, Shimizu M, Yan GX, Sicouri S, Weissenburger J, Nesterenko W, Burashnikov A, Di Diego J, Saffitz J, Thomas GP: The Mcell: its contribution to theECGand to normal and abnormal electrical function of the heart J Cardiovasc Electrophysiol 1999, 10:1124-1152.

38. Malik M, Färbom P, Batchvarov V, Hnatkova K, Camm AJ: Relation between QT and RR intervals is highly individual among healthy subjects: Implications for heart rate correction on the QT interval. Heart 2002 87:220-228.

39. Valentinuzzi ME: A mathematical model of the electrocardiographic QT-RR relationship. Med Biol Eng Comput $1971,9: 255-261$.

doi:10.1186/1742-4682-9-15

Cite this article as: Bonomini et al:: The allometric model in chronic myocardial infarction. Theoretical Biology and Medical Modelling 2012 9:15.

\section{Submit your next manuscript to BioMed Central and take full advantage of:}

- Convenient online submission

- Thorough peer review

- No space constraints or color figure charges

- Immediate publication on acceptance

- Inclusion in PubMed, CAS, Scopus and Google Scholar

- Research which is freely available for redistribution 